\title{
Article \\ The Influence of Adjuvant Chemotherapy Dose Intensity on Five-Year Outcomes in Resected Colon Cancer: A Single Centre Retrospective Analysis
}

\author{
Suganija Lakkunarajah ${ }^{1}$, Daniel A. Breadner ${ }^{2,3, *}$, Hanbo Zhang ${ }^{4}$, Ellen Yamanaka ${ }^{3}$, Andrew Warner ${ }^{3}$ and \\ Stephen Welch 2,3 \\ 1 Department of Medicine, Schulich School of Medicine \& Dentistry, Western University, \\ London, ON N6A 5A5, Canada; suganija.lakkunarajah@lhsc.on.ca \\ 2 Department of Oncology, Schulich School of Medicine \& Dentistry, Western University, \\ London, ON N6A 5W9, Canada; stephen.welch@lhsc.on.ca \\ 3 London Regional Cancer Program, London Health Sciences Centre, Victoria Hospital, \\ London, ON N6A 5W9, Canada; Ellen.yamanaka@lhsc.on.ca (E.Y.); Andrew.warner@lhsc.on.ca (A.W.) \\ 4 Department of Internal Medicine, Section of Hematology and Oncology, CancerCare Manitoba, \\ Winnipeg, MB R3E 0V9, Canada; hzhang2@cancercare.mb.ca \\ * Correspondence: daniel.breadner@lhsc.on.ca
}

Citation: Lakkunarajah, S.; Breadner, D.A.; Zhang, H.; Yamanaka, E.; Warner, A.; Welch, S. The Influence of Adjuvant Chemotherapy Dose Intensity on Five-Year Outcomes in Resected Colon Cancer: A Single Centre Retrospective Analysis. Curr. Oncol. 2021, 28, 4031-4041. https://doi.org/10.3390/ curroncol28050342

Received: 15 June 2021

Accepted: 28 September 2021

Published: 9 October 2021

Publisher's Note: MDPI stays neutral with regard to jurisdictional claims in published maps and institutional affiliations.

Copyright: (c) 2021 by the authors. Licensee MDPI, Basel, Switzerland. This article is an open access article distributed under the terms and conditions of the Creative Commons Attribution (CC BY) license (https:/ / creativecommons.org/licenses/by/ $4.0 /)$.

\begin{abstract}
There is evidence that achieving a dose intensity $>80 \%$ in adjuvant colon cancer treatment improves survival. In total, 192 consecutive patients with resected stage III and high-risk stage II colon cancer that received adjuvant chemotherapy were retrospectively analyzed. Patients who received at least 6 weeks of adjuvant therapy were included. The primary objective was to assess the influence of dose index (DI) and relative dose intensity (RDI) on DFS and OS at 3 and 5 years in patients receiving fluorouracil-based doublet therapy with oxaliplatin (FOLFOX) (5-FU and oxaliplatin assessed separately), or capecitabine monotherapy. In the capecitabine group, DFS rates for 3 and 5 years were $66.7 \%$ and $57.6 \%$, respectively, while OS rates were $80.3 \%$ and $66.7 \%$, respectively. Those who received FOLFOX had DFS rates of $76.9 \%$ and $71.2 \%$ at 3 and 5 years, respectively. OS rates were $86.4 \%$ and $76.7 \%$ at 3 and 5 years, respectively. Median RDI was $73.8 \%$ for capecitabine and $76.3 \%$ and $85.6 \%$ for the oxaliplatin and 5-FU components respectively. Based on a multivariate analysis in patients receiving FOLFOX, those with an oxaliplatin DI $>80 \%$ had improvements in DFS and OS compared to those with an oxaliplatin DI of $\leq 80 \%$. Otherwise, there was no significant difference in DFS or OS when comparing patients who achieved an RDI or a DI of above versus below $80 \%$ in the patients receiving adjuvant chemotherapy for resected colon cancer.
\end{abstract}

Keywords: dose index; relative dose intensity; colon cancer

\section{Introduction}

Colorectal cancer (CRC) remains the second leading cause of cancer death in developed countries, despite the implementation of early detection and screening programs [1-3]. Patients with stage III colon cancer (CC) have benefited significantly from clinical trials, which have substantially reduced the risk of recurrence in this vulnerable group. A few notable changes include 5-fluorouracil (5-FU) based adjuvant chemotherapy, then the addition of oxaliplatin chemotherapy, and the resection of an increased number of lymph nodes for a more complete excision with more accurate staging [4-6]. There is substantial research both published and ongoing regarding the timing, duration and choice of different chemotherapy regimens in this population.

In 2018, the International Duration Evaluation of Adjuvant Chemotherapy (IDEA) collaboration reported its findings looking at three versus six months of adjuvant folinic acid + 5-FU + oxaliplatin (FOLFOX) or capecitabine and oxaliplatin (CAPOX) in patients with resected stage III CC from 12 different countries. They reported 3 months was not 
non-inferior to 6 months of adjuvant treatment, except in patients with non-T4 and non-N2 disease treated with CAPOX. Considering that three months of adjuvant treatment is sufficient in some populations, it warrants a review of the influence of dose index (DI) and relative dose intensity (RDI) of oxaliplatin-based chemotherapy on disease-free survival (DFS) and overall survival (OS).

Oxaliplatin has improved outcomes when compared to 5-FU/leucovorin alone in both adjuvant stage III CC and in metastatic CRC [4,7]. Oxaliplatin induced sensory neuropathy are common with FOLFOX and CAPOX and can lead to reduced quality of life, dose reduction, delays, or discontinuation of treatment [8]. Oxaliplatin toxicity increases after three months, especially in the elderly, and the degree of its benefits thereafter varies [9-11].

This paper examines the impact of DI and RDI on efficacy outcomes of a populationbased cohort of patients with resected stage III CC receiving adjuvant chemotherapy. The primary objective was to assess the influence of DI on DFS and OS for capecitabine and separately for the 5-FU and oxaliplatin components of FOLFOX. Additionally, the influence of RDI and rates of dose delays, dose reductions and 5-FU associated cardiac toxicity were examined.

\section{Methods}

At a Canadian academic cancer centre, all patients who were seen in consultation for resected stage III CC and received adjuvant chemotherapy between 2006 and 2011 were retrospectively analyzed. Patients were excluded if they developed metastasis within two months of surgery, as these patients were felt to have occult metastatic disease. Patients were excluded from analysis if they received neoadjuvant chemotherapy and/or adjuvant radiation, or if they were determined to have rectal cancer. Patients were only included in the analysis if they received at least three cycles of FOLFOX or two cycles of capecitabine, as it is previously reported that patients with stage III CC who receive limited chemotherapy do not have improved outcomes compared to surgery alone [10,12].

Analysis of DI and RDI was restricted to patients who received FOLFOX or capecitabine as adjuvant therapy. Patients who switched from FOLFOX to capecitabine had their dose of capecitabine converted to its 5-FU equivalent percentage, and were included in the analysis as patients on FOLFOX. Patients switched from FOLFOX to raltitrexed and oxaliplatin were considered to have stopped therapy and further oxaliplatin was not included in the calculation of oxaliplatin DI and RDI. Patients treated with alternative regimens were not included in analysis, as summarized in Figure 1.

DI and RDI calculations were completed using standard doses for FOLFOX, bolus 5 -FU at $400 \mathrm{mg} / \mathrm{m}^{2}, 46 \mathrm{~h} 5$-FU infusion at $2400 \mathrm{mg} / \mathrm{m}^{2}$, and oxaliplatin at $85 \mathrm{mg} / \mathrm{m}^{2}$. The capecitabine ideal dose was $1250 \mathrm{mg} / \mathrm{m}^{2}$ orally twice daily, although there is evidence supporting that $1000 \mathrm{mg} / \mathrm{m}^{2}$ orally twice daily is a safer and effective dose in elderly or frail patients, and this dose is commonly used in clinical practice $[13,14]$. The higher dose was selected as some younger or fit patients elected for capecitabine over intravenous combination chemotherapy where the target dose is $1250 \mathrm{mg} / \mathrm{m}^{2}$ orally twice daily.

\section{Statistical Analysis}

DI and RDI were both calculated based on the definitions established by Hryniuk [15,16]. Patients were classified as having a dose reduction if they received less than $90 \%$ of the ideal dose during any cycle of their chemotherapy. Dose delays were defined as receiving a cycle of chemotherapy greater than 13 days after the regimen's scheduled date for either FOLFOX or capecitabine. Descriptive statistics were generated for baseline patient characteristics for all patients and stratified by chemotherapy regimen, compared using the chi-square test, Fisher's exact test, two-sample t-test or Wilcoxon rank sum test as appropriate. DFS and OS were calculated from the date of surgery to the date of recurrence (DFS only), death due to any cause, or date of last follow-up, whichever occurs first. Kaplan-Meier estimates were generated for DFS and OS, stratified by DI or RDI ( $\leq 80 \%$ vs. $>80 \%)$ of capecitabine, 5-FU, and oxaliplatin, and compared using the log-rank test. As a post hoc 
sensitivity analysis, multivariable Cox proportional hazards regression was performed for DFS and OS adjusted for: chemotherapy regimen (one of: chemotherapy regimen, DI or RDI of capecitabine, 5-FU and oxaliplatin), gender, age, any comorbidity, pathological T stage and $\mathrm{N}$ stage. All statistical analyses were performed using SAS version 9.4 software (SAS Institute, Cary, NC, USA) with two-sided statistical testing at the 0.05 significance level.

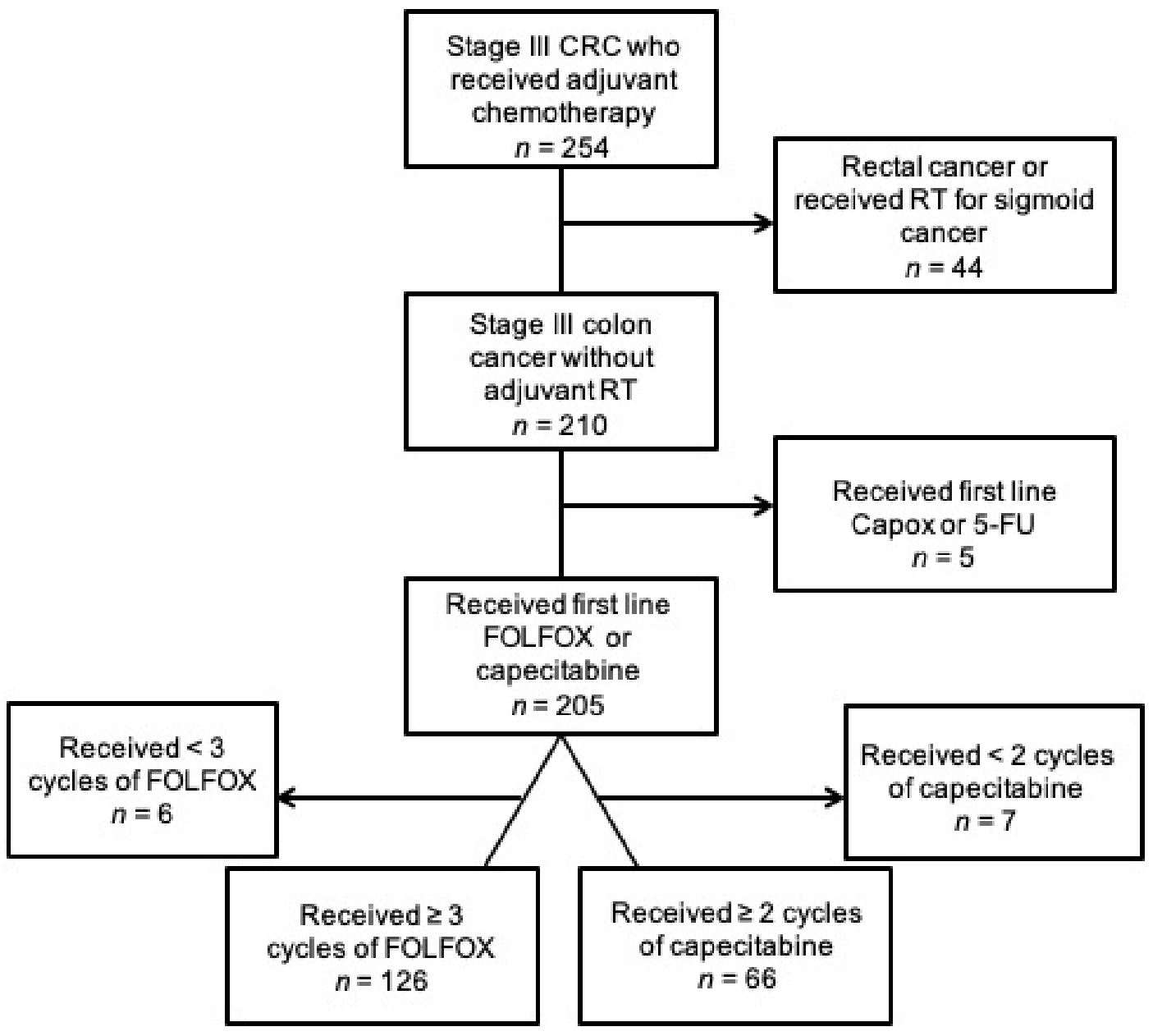

Figure 1. Summary of patient selection.

\section{Results}

\subsection{Patient Characteristics}

Between 2008 and 2012, 254 patients received adjuvant chemotherapy for stage III CRC. Of this group of patients, 192 CC patients received at least a quarter of the planned cycles of FOLFOX $(n=126)$ or capecitabine $(n=66)$ without any adjuvant radiation therapy. Mean \pm SD age was $74.5 \pm 6.8$ years for those who received capecitabine, which was significantly higher than $62.5 \pm 8.3$ years for those who received FOLFOX $(p<0.001)$. Patients receiving capecitabine also had a significantly higher proportion of having at least one comorbidity compared to FOLFOX $(89.4 \%$ vs. $77.0 \%, p=0.036)$, as shown in Table 1 . Dosing changes were more common for patients on FOLFOX compared to capecitabine with $77.0 \%$ vs. $60.6 \%$ requiring at least 1 dose reduction ( $p=0.017), 60.3 \%$ vs. $45.5 \%$ had dose delays $(p=0.049)$, while $63.5 \%$ vs. $68.2 \%$ completed all planned cycles of therapy $(p=0.517)$. A vast majority of patients initiated therapy with dose intensity of at least $80 \%$, specifically 121 of 126 patients receiving oxaliplatin started treatment above that threshold. Cardiac toxicity (acute coronary syndrome or coronary vasospasm) occurred in 13 patients $(6.8 \%)$. 
Table 1. Baseline patient characteristics $(n=192)$.

\begin{tabular}{|c|c|c|c|c|}
\hline Characteristic & $\begin{array}{l}\text { All Patients } \\
\quad(n=192)\end{array}$ & $\begin{array}{l}\text { Capecitabine } \\
\quad(n=66)\end{array}$ & $\begin{array}{l}\text { FOLFOX } \\
(n=126)\end{array}$ & $p$-Value \\
\hline \multicolumn{5}{|l|}{ Gender- $n(\%)$} \\
\hline Male & $98(51.0)$ & $36(54.6)$ & $62(49.2)$ & \multirow[t]{2}{*}{0.482} \\
\hline Female & $94(49.0)$ & $30(45.5)$ & $64(50.8)$ & \\
\hline Age-median (IQR) & $67.7(60.5,73.0)$ & $75.1(71.7,78.9)$ & $63.8(57.5,69.1)$ & $<0.001$ \\
\hline $\begin{array}{l}\text { Resection to discharge } \\
\text { (days)-median (IQR) }\end{array}$ & $6(5,8)$ & $7(6,9)$ & $6(4,8)$ & 0.004 \\
\hline $\begin{array}{l}\text { Post-operative hospital } \\
\text { re-admission }-n(\%)\end{array}$ & $14(7.3)$ & $8(12.1)$ & $6(4.8)$ & 0.080 \\
\hline $\begin{array}{l}\text { Surgery to medical oncology referral } \\
\text { consult (days)—-median (IQR) }\end{array}$ & $21(14,30)$ & $23(15,31)$ & $21(13,28)$ & 0.226 \\
\hline Comorbidity: any-n (\%) & $156(81.3)$ & $59(89.4)$ & $97(77.0)$ & 0.036 \\
\hline Perioperative complications- $n(\%)$ & $49(25.5)$ & $25(37.9)$ & $24(19.1)$ & 0.005 \\
\hline \multicolumn{5}{|l|}{ Pathological T stage $-n(\%)$} \\
\hline T0 & $1(0.5)$ & $0(0)$ & $1(0.8)$ & \multirow{5}{*}{0.803} \\
\hline $\mathrm{T} 1$ & $7(3.7)$ & $2(3.0)$ & $5(4.0)$ & \\
\hline $\mathrm{T} 2$ & $12(6.3)$ & $3(4.6)$ & $9(7.1)$ & \\
\hline T3 & $93(48.4)$ & $36(54.6)$ & $57(45.2)$ & \\
\hline $\mathrm{T} 4$ & $79(41.2)$ & $25(37.9)$ & $54(42.9)$ & \\
\hline \multicolumn{5}{|l|}{ Pathological N stage $-n(\%)$} \\
\hline N0 & $30(15.6)$ & $12(18.2)$ & $18(14.3)$ & \multirow{3}{*}{0.605} \\
\hline N1 & $111(57.8)$ & $39(59.1)$ & $72(57.1)$ & \\
\hline $\mathrm{N} 2$ & $51(26.6)$ & $15(22.7)$ & $36(28.6)$ & \\
\hline \multicolumn{5}{|l|}{ Status- $n(\%)$} \\
\hline Alive with disease & $5(2.6)$ & $2(3.0)$ & $3(2.4)$ & \multirow{4}{*}{0.083} \\
\hline Alive without disease & $129(67.2)$ & $38(57.6)$ & $91(72.2)$ & \\
\hline Death with disease & $43(22.4)$ & $17(25.8)$ & $26(20.6)$ & \\
\hline Death without disease & $15(7.8)$ & $9(13.6)$ & $6(4.8)$ & \\
\hline Death-n $(\%)$ & $58(30.2)$ & $26(39.4)$ & $32(25.4)$ & 0.045 \\
\hline Recurrence- $n(\%)$ & $59(30.7)$ & $23(34.9)$ & $36(28.6)$ & 0.371 \\
\hline $\begin{array}{l}\text { Median follow-up (years)-median } \\
\qquad(95 \% \mathrm{CI})\end{array}$ & $5.74(5.52,5.96)$ & $5.79(5.46,6.08)$ & $5.74(5.45,6.01)$ & 0.758 \\
\hline
\end{tabular}

$\mathrm{IQR}=$ Interquartile range.

The median DI for capecitabine was $72.5 \%$ and the median DI for patients receiving FOLFOX was $77.0 \%$ and $89.4 \%$ for oxaliplatin and 5-FU, respectively. Similarly, the median RDI for capecitabine was $73.8 \%$ and the median DI for patients receiving FOLFOX was $76.3 \%$ and $85.6 \%$ for oxaliplatin and 5-FU, respectively.

DFS and OS data were available at 3-years and 5-years for the vast majority of patients. One patient was lost to follow-up prior to 3 years post-surgery due to moving out of country, and 10 patients were lost to follow-up prior to 5 years due to either not yet reached, or not had medical follow-up visits despite reaching 5 years post-surgery. Median follow-up was 5.8 years for patients receiving capecitabine and 5.7 years for patients receiving FOLFOX.

\subsection{Disease-Free Survival}

Kaplan-Meier plots for DFS stratified by DI ( $\leq 80 \%$ vs. $>80 \%$ ) of capecitabine, 5-FU, and oxaliplatin are shown in Figures 2 and 3 and corresponding estimates summarized in Table 2. For patients who received capecitabine, 3- and 5-year DFS was $65.9 \%$ and $56.8 \%$ for DI $\leq 80 \%$ and $68.2 \%$ and $59.1 \%$ for DI $>80 \%$, however there was no significant difference ( $\log$-rank $p=0.951$ ). For patients who received FOLFOX, 3- and 5-year DFS was $80.3 \%$ and $67.4 \%$ for 5 -FU DI $\leq 80 \%$ and $75.3 \%$ and $72.9 \%$ for 5 -FU DI $>80 \%$, however this was 
not significant $(\log$-rank $p=0.753)$. Similarly, 3- and 5-year DFS was $77.4 \%$ and $68.1 \%$ for oxaliplatin DI $\leq 80 \%$ and $76.3 \%$ and $74.6 \%$ for oxaliplatin DI $>80 \%$, however this was not significant ( $\log$-rank $p=0.544)$. Overall, there was also no significant difference comparing all 5-FU and oxaliplatin DI groups and no DFS improvement was observed for patients receiving a DI $>80 \%$ (log-rank $p=0.925)$. Results for DFS stratified by RDI $(\leq 80 \%$ vs. $>80 \%$ ) of capecitabine, 5 -FU, and oxaliplatin were consistent with those for DI and are shown in Supplemental Figures S1 and S2 and Table S1.

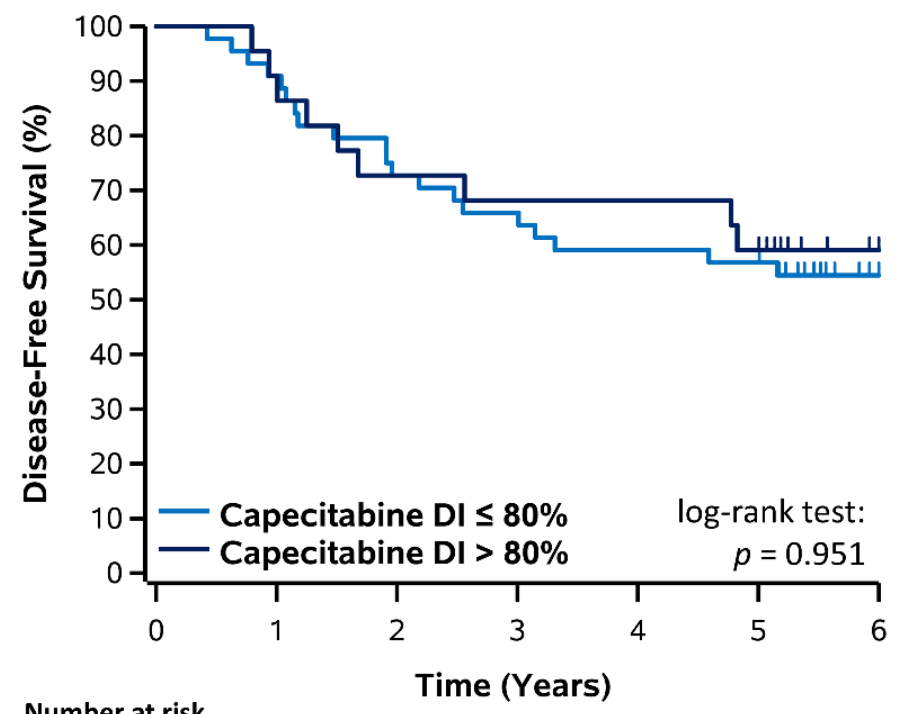

Number at risk

$\begin{array}{lllllll}44 & 40 & 32 & 29 & 26 & 25 & 9 \\ 22 & 20 & 16 & 15 & 15 & 13 & 5\end{array}$

Figure 2. Kaplan-Meier plot of DFS stratified by DI of capecitabine in patients receiving capecitabine.

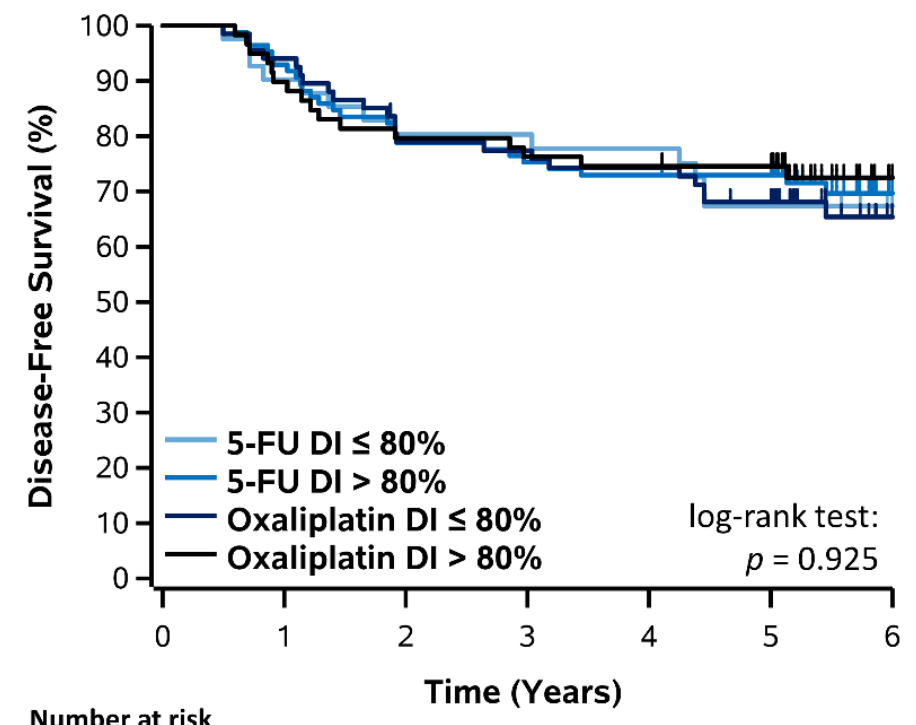

Number at risk

$\begin{array}{lllllll}41 & 37 & 31 & 31 & 30 & 25 & 9 \\ 85 & 79 & 67 & 64 & 62 & 61 & 25 \\ 67 & 63 & 51 & 50 & 48 & 43 & 17 \\ 59 & 53 & 47 & 45 & 44 & 43 & 17\end{array}$

Figure 3. Kaplan-Meier plot of DFS stratified by DI of 5-FU and oxaliplatin in patients receiving FOLFOX. 
Table 2. The 3 and 5-year DFS and OS stratified by DI of capecitabine, 5-FU and oxaliplatin.

\begin{tabular}{|c|c|c|c|c|c|}
\hline \multirow[b]{2}{*}{ Dose Intensity } & \multicolumn{2}{|c|}{ 3-Year DFS (\%) } & \multicolumn{2}{|c|}{ 5-Year DFS (\%) } & \multirow[t]{2}{*}{ Log-Rank $p$-Value } \\
\hline & $\leq 80 \%$ & $>80 \%$ & $\leq 80 \%$ & $>80 \%$ & \\
\hline Capecitabine & $65.9 \%$ & $68.2 \%$ & $56.8 \%$ & $59.1 \%$ & 0.951 \\
\hline 5-FU & $80.3 \%$ & $75.3 \%$ & $67.4 \%$ & $72.9 \%$ & 0.753 \\
\hline \multirow[t]{2}{*}{ Oxaliplatin } & $77.4 \%$ & $76.3 \%$ & $68.1 \%$ & $74.6 \%$ & 0.544 \\
\hline & \multicolumn{2}{|c|}{ 3-Year OS (\%) } & \multicolumn{2}{|c|}{ 5-Year OS (\%) } & Log-Rank $p$-Value \\
\hline Dose Intensity & $\leq 80 \%$ & $>80 \%$ & $\leq 80 \%$ & $>80 \%$ & \\
\hline Capecitabine & $81.8 \%$ & $77.3 \%$ & $68.2 \%$ & $63.6 \%$ & 0.881 \\
\hline 5-FU & $87.7 \%$ & $85.9 \%$ & $74.8 \%$ & $77.6 \%$ & 0.700 \\
\hline Oxaliplatin & $87.8 \%$ & $84.7 \%$ & $75.5 \%$ & $77.9 \%$ & 0.480 \\
\hline
\end{tabular}

Multivariable Cox proportional hazards regression for DFS (adjusting for gender, age, any comorbidity, pathological $\mathrm{T}$ stage and $\mathrm{N}$ stage) similarly identified no significant differences for patients with capecitabine DI $>80 \%$ compared to $\leq 80 \%$ (hazard ratio [HR]: 1.12; $95 \%$ confidence interval [CI]: $0.50-2.51 ; p=0.787$ ) and 5 -FU DI $>80 \%$ compared to $\leq 80 \%$ (HR: $0.50 ; 95 \%$ CI: $0.24-1.02 ; p=0.058$ ). However, patients with oxaliplatin DI $>80 \%$ had significantly improved DFS compared to $\leq 80 \%$ (HR: 0.36 ; $95 \%$ CI: $0.17-0.75 ; p=0.006$ ). In contrast, no significant differences were observed for RDI $>80 \%$ compared to $\leq 80 \%$ for each of capecitabine, 5-FU and oxaliplatin.

\subsection{Overall Survival}

Similarly, Kaplan-Meier plots for OS stratified by DI ( $\leq 80 \%$ vs. $>80 \%$ ) of capecitabine, 5-FU, and oxaliplatin are shown in Figures 4 and 5 and corresponding estimates also summarized in Table 2. For patients who received capecitabine, 3- and 5-year OS was $81.8 \%$ and $68.2 \%$ for DI $\leq 80 \%$ and $77.3 \%$ and $63.6 \%$ for DI $>80 \%$, however there was no significant difference (log-rank $p=0.881$ ). For patients who received FOLFOX, 3 - and 5-year OS was $87.7 \%$ and $74.8 \%$ for 5 -FU DI $\leq 80 \%$ and $85.9 \%$ and $77.6 \%$ for 5 -FU DI $>80 \%$, however this was not significant ( $\log -\operatorname{rank} p=0.700)$. Similarly, 3- and 5-year OS was $87.8 \%$ and $75.5 \%$ for oxaliplatin DI $\leq 80 \%$ and $84.7 \%$ and $77.9 \%$ for oxaliplatin DI $>80 \%$, however this was not significant $(\log$-rank $p=0.480)$. Overall, there was also no significant difference comparing all 5-FU and oxaliplatin DI groups and no OS improvement was observed for patients receiving a DI $>80 \%$ (log-rank $p=0.885)$. Results for OS stratified by RDI ( $\leq 80 \%$ vs. $>80 \%$ ) of capecitabine, $5-\mathrm{FU}$, and oxaliplatin were consistent with those for DI and are shown in Supplemental Figures S3 and S4 and Table S1.

Multivariable Cox proportional hazards regression for OS also identified no significant differences for patients with capecitabine DI $>80 \%$ compared to $\leq 80 \%$ (HR: 1.21 ; 95\% CI: $0.51-2.90 ; p=0.667$ ) and 5-FU DI $>80 \%$ compared to $\leq 80 \%$ (HR: $0.46 ; 95 \%$ CI: $0.21-1.01 ; p=0.054)$. Patients with oxaliplatin DI $>80 \%$ also had significantly improved OS compared to $\leq 80 \%$ (HR: $0.33 ; 95 \%$ CI: $0.14-0.74 ; p=0.007$ ). In contrast, no significant differences were observed for RDI $>80 \%$ compared to $\leq 80 \%$ for each of capecitabine, 5-FU and oxaliplatin. 


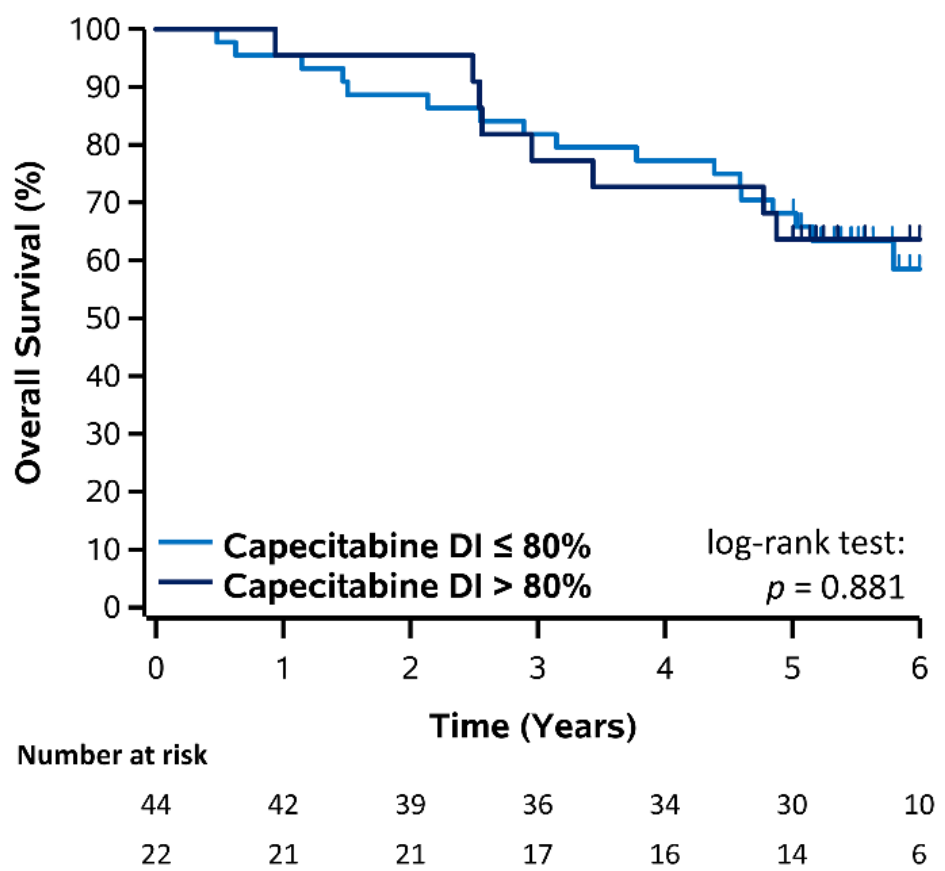

Figure 4. Kaplan-Meier plot of OS stratified by DI of capecitabine in patients receiving capecitabine.

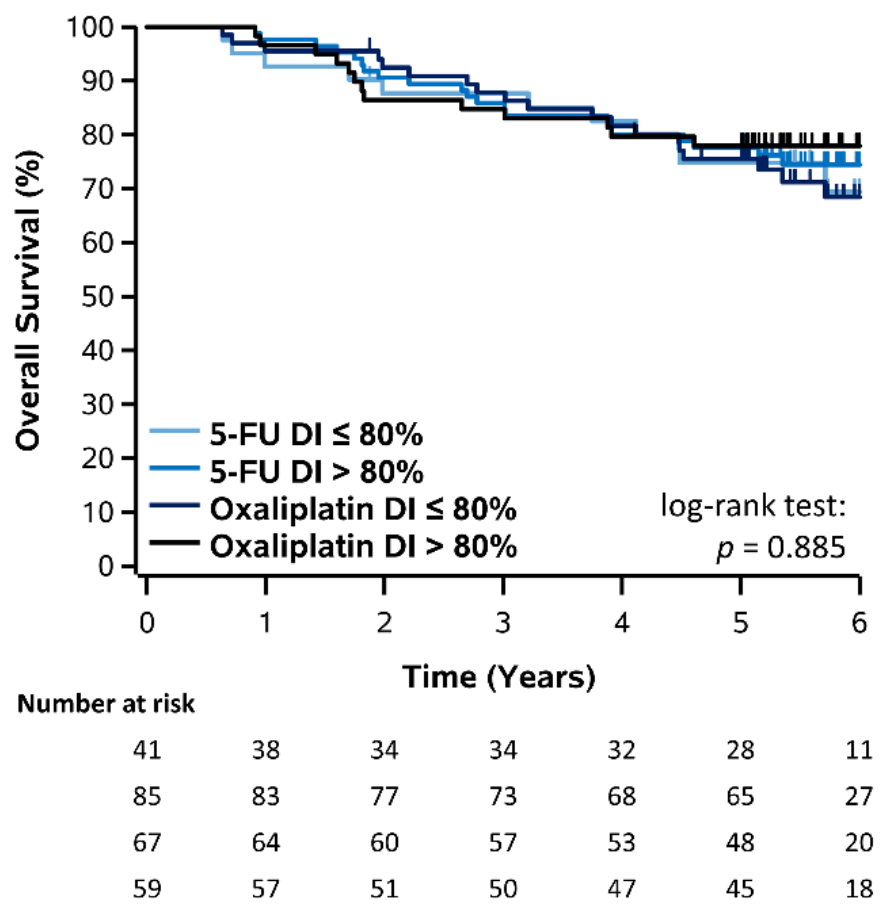

Figure 5. Kaplan-Meier plot of OS stratified by DI of 5-FU and oxaliplatin in patients receiving FOLFOX.

\section{Discussion}

This single-centre retrospective study reports no significant difference in survival outcomes at 3 or 5 years based on DI or RDI for capecitabine, as monotherapy, or the 5-FU component of FOLFOX in the adjuvant setting for stage III CRC. Oxaliplatin RDI did not significantly affect outcomes. However, there was an improvement in DFS and OS in patients with an oxaliplatin DI above $80 \%$ compared to those with a DI of less than $80 \%$, based on multivariable analysis.

Much variability exists in the literature as to how to define and calculate DI (also referred to as dose intensity). The current study selected the definition proposed by 
Hryniuk, which calculates the actual dose received against the ideal dose based on the regimen [15]. Similarly, there are multiple definitions for calculating RDI [15,17], however the current study selected the definition proposed by Hryniuk. All definitions calculate RDI based on the ideal dose over the target delivery time, however there is a variation on whether early cessation is incorporated, although it always leads to a reduction in DI. The DI definition used in the current study does not take into consideration the time taken to complete the treatment and may not accurately represent those who had dose delays. This investigation emphasized DI over RDI results as there is good evidence that a patient receiving a full dose of FOLFOX or CAPOX for 3 months and then stopping treatment would have a different level of benefit than a patient receiving a 50\% dose of FOLFOX or CAPOX for 6 months, and completing on schedule. Both scenarios may translate to an RDI of $50 \%$, depending on the definition used.

RDI has been shown to have an impact on outcomes in a number of cancers, including breast, lung, lymphoma, and CRC [16,18-22]. In the metastatic CRC setting, Nakayama reported an RDI of greater than $80 \%$ of irinotecan in folinic acid + 5-FU + irinotecan (FOLFIRI) based chemotherapy was associated with a significant improvement in response rate (RR), disease control rate (DCR), progression-free survival (PFS), and OS; while an RDI of greater than $79 \%$ of oxaliplatin in FOLFOX demonstrated a significant improvement in DCR in a study population of only 30 patients [16]. In stage III CC patients, Morris reported failing to complete more than three cycles of adjuvant therapy led to outcomes inferior to surgery alone, while patients completing four or more cycles had superior outcomes [12]. Two additional studies by Aspinall and Ho revealed an RDI of greater than $70 \%$ was associated with improved outcomes in stage III CC [23,24]. Aspinall noted a 5 -year OS improvement of $15.8 \%$ and a 3 -year DFS increase of $13.4 \%$ when RDI was greater than $70 \%$. However, Ho's abstract was only reporting on capecitabine monotherapy, and Aspinall's analysis, although well powered, only included American veterans, so neither study reported outcomes for oxaliplatin based chemotherapy in a general population.

There is now evidence that extending adjuvant chemotherapy beyond 3 months is not beneficial in some circumstances and can lead to increased toxicity $[25,26]$. In practice, physicians try to balance toxicity with the objective of reducing CC recurrence risk. This is especially true with oxaliplatin chemotherapy, which carries a considerable risk of acute and chronic peripheral neuropathy. This retrospective analysis seeks to inform patients and physicians of the trade-off in CC recurrence risk with toxicity and safety in patients requiring a dose reduction.

The two regimens assessed in this analysis were FOLFOX and capecitabine monotherapy, as CAPOX was not reimbursed during the examined period. 5-FU and oxaliplatin doublet is considered the standard of care for adjuvant therapy in high-risk stage II or stage III CC, in medically fit patients. This report reveals a DFS and OS benefit in patients receiving at least $80 \%$ of the intended oxaliplatin in a population of 126 patients receiving FOLFOX adjuvant chemotherapy for $\mathrm{CC}$. There were no other differences in clinical outcomes based on oxaliplatin RDI or 5-FU DI or RDI in this group of patients receiving FOLFOX. Considering the growing evidence that 3 months of adjuvant doublet chemotherapy is equally as effective as 6 months, in certain circumstances, perhaps the more poignant question is to examine the influence of DI or RDI in the first three months of treatment and the time from surgery to initiating adjuvant chemotherapy. A larger population would also allow subgroup analyses in those with certain risk factors, such as pathological $\mathrm{T} 4$ or $\mathrm{N} 2$ resected disease.

The 66 patients receiving capecitabine monotherapy in this study were significantly older than the FOLFOX population. This was expected as monotherapy is typically reserved for older or frail patients or those with contraindications to oxaliplatin [14]. This study did not include a comparison between doublet and monotherapy, as it is well established that capecitabine monotherapy is inferior to oxaliplatin-based regimens [27]. This study contained a subset of patients receiving capecitabine monotherapy and there is limited data on dose intensity in the adjuvant setting for older or frail patients, and data on monotherapy 
predating the widespread use of oxaliplatin does not generally apply. Neugut et al. had examined patients $\geq 65$ years old receiving 5 -FU based chemotherapy and observed that early cessation of treatment was associated with a doubling of mortality rates compared to patients completing 6 months of treatment [10]. In this retrospective observational study we report no significant difference in survival outcomes when a DI of less than $80 \%$ is achieved, which is reassuring for patients and clinicians when capecitabine dose reductions and/or early cessation are needed.

There are several limitations in this study; most particularly it is a retrospective single centre analysis based on a smaller population size. Therefore, this study may not have been sufficiently powered to detect the influence of dose intensity on recurrence. A clear strength is that three-year DFS and OS data were available on all but one patient, and a vast majority of patients had five-year data available. Collaboration with other Canadian institutions would allow for a more sufficiently powered examination of the effect of DI on 5-year OS and DFS in patients with resected CC and validate the results of the current study.

\section{Conclusions}

In 126 patients with resected high-risk stage II and stage III CC, receiving adjuvant FOLFOX, patients receiving at least $80 \%$ of the ideal dose of oxaliplatin had improvements in DFS and OS, compared to those with a DI of less than $80 \%$. Oxaliplatin RDI did significantly influence DFS or OS. There was no difference in survival outcomes in patients receiving FOLFOX with a 5-FU DI or RDI of at least $80 \%$ compared to those with a 5-FU DI or RDI of less than $80 \%$. In 66 patients receiving adjuvant capecitabine monotherapy there were no differences in recurrence or survival outcomes based on DI or RDI of at least $80 \%$ compared to those with a 5-FU DI or RDI of less than $80 \%$. Considering recent evidence challenging the need for 6 months of adjuvant chemotherapy for some patients, examining the influence of DI and RDI in the first three months of adjuvant therapy would be more applicable, in certain populations. Pooling data from multiple institutions is needed to better evaluate the influence of DI and RDI on adjuvant CC survival outcomes.

Supplementary Materials: The following are available online at https:/ / www.mdpi.com/article/ 10.3390/curroncol28050342/s1, Table S1: The 3 and 5-year DFS and OS are reported for those who achieved greater than $80 \%$ RDI for the respective therapeutic versus those who did not exceed $80 \%$ RDI, Figure S1: Kaplan-Meier plot of DFS stratified by RDI of capecitabine in patients receiving capecitabine, Figure S2: Kaplan-Meier plot of DFS stratified by RDI of 5-FU and oxaliplatin in patients receiving FOLFOX, Figure S3: Kaplan-Meier plot of OS stratified by RDI of capecitabine in patients receiving capecitabine, Figure S4: Kaplan-Meier plot of OS stratified by RDI of 5-FU and oxaliplatin in patients receiving FOLFOX.

Author Contributions: Conceptualization, D.A.B. and S.W.; methodology, A.W., E.Y., D.A.B.; software, E.Y. and A.W.; formal analysis, E.Y. and A.W.; investigation, H.Z. and D.A.B.; writing-original draft preparation, D.A.B. and S.L.; writing-review and editing, A.W., S.W., H.Z., E.Y.; supervision, S.W. and D.A.B.; funding acquisition, S.W. All authors have read and agreed to the published version of the manuscript.

Funding: This research received no external funding.

Institutional Review Board Statement: The study was conducted according to the guidelines of the Declaration of Helsinki, and approved by the Institutional Review Board (or Ethics Committee) of Western University (WREM\# 102843).

Informed Consent Statement: Patient consent was waived as this was a retrospective analysis.

Data Availability Statement: Data from this study are available to researchers through a data access process.

Conflicts of Interest: The authors declare no conflict of interest. 


\section{References}

1. Committee CCSA. Canadian Cancer Statistics 2019; Canadian Cancer Society: Toronto, ON, USA, 2019.

2. Mph, K.D.M.; Nogueira, L.; Mariotto, A.B.; Rowland, J.H.; Yabroff, K.R.; Alfano, C.M.; Jemal, A.; Kramer, J.L.; Siegel, R.L. Cancer treatment and survivorship statistics, 2019. CA Cancer J. Clin. 2019, 69, 363-385.

3. National Cancer Institute. SEER Cancer Statistics Factsheets: Colon and Rectum Cancer. 2013. Available online: http://seer. cancer.gov/statfacts/html/colorect.html (accessed on 12 June 2021).

4. André, T.; Boni, C.; Mounedji-Boudiaf, L.; Navarro, M.; Tabernero, J.; Hickish, T.; Topham, C.; Zaninelli, M.; Clingan, P.; Bridgewater, J.; et al. Oxaliplatin, Fluorouracil, and Leucovorin as Adjuvant Treatment for Colon Cancer. N. Engl. J. Med. 2004, 350, 2343-2351. [CrossRef] [PubMed]

5. Investigators, I. Efficacy of adjuvant fluorouracil and folinic acid in colon cancer. Lancet 1995, 345, 939-944.

6. Wong, S.L.; Ji, H.; Hollenbeck, B.K.; Morris, A.M.; Baser, O.; Birkmeyer, J.D. Hospital Lymph Node Examination Rates and Survival after Resection for Colon Cancer. JAMA 2007, 298, 2149-2154. [CrossRef]

7. de Gramont, A.D.; Figer, A.; Seymour, M.; Homerin, M.; Hmissi, A.; Cassidy, J.; Boni, C.; Cortes-Funes, H.; Cervantes, A.; Freyer, G. Leucovorin and fluorouracil with or without oxaliplatin as first-line treatment in advanced colorectal cancer. J. Clin. Oncol. 2000, 18, 2938-2947. [CrossRef]

8. Soveri, L.; Lamminmäki, A.; Hänninen, U.; Karhunen, M.; Bono, P.; Osterlund, P. Long-term neuropathy and quality of life in colorectal cancer patients treated with oxaliplatin containing adjuvant chemotherapy. Acta Oncol. 2019, 58, 398-406. [CrossRef]

9. Goldberg, R.M.; Tabah-Fisch, I.; Bleiberg, H.; de Gramont, A.; Tournigand, C.; Andre, T.; Rothenberg, M.L.; Green, E.; Sargent, D.J. Pooled Analysis of Safety and Efficacy of Oxaliplatin Plus Fluorouracil/Leucovorin Administered Bimonthly in Elderly Patients with Colorectal Cancer. J. Clin. Oncol. 2006, 24, 4085-4091. [CrossRef]

10. Neugut, A.I.; Matasar, M.; Wang, X.; McBride, R.; Jacobson, J.S.; Tsai, W.-Y.; Grann, V.R.; Hershman, D.L. Duration of Adjuvant Chemotherapy for Colon Cancer and Survival among the Elderly. J. Clin. Oncol. 2006, 24, 2368-2375. [CrossRef]

11. Tournigand, C.; Cervantes, A.; Figer, A.; Lledo, G.; Flesch, M.; Buyse, M.; Mineur, L.; Carola, E.; Etienne, P.-L.; Rivera, F.; et al. OPTIMOX1: A Randomized Study of FOLFOX4 or FOLFOX7 with Oxaliplatin in a Stop-and-Go Fashion in Advanced Colorectal Cancer-A GERCOR Study. J. Clin. Oncol. 2006, 24, 394-400. [CrossRef]

12. Morris, M.; Platell, C.; Fritschi, L.; Iacopetta, B. Failure to complete adjuvant chemotherapy is associated with adverse survival in stage III colon cancer patients. Br. J. Cancer 2007, 96, 701-707. [CrossRef]

13. Vincent, M.D.; Breadner, D.; Soulieres, D.; Kerr, I.G.; Sanatani, M.; Kocha, W.; Klimo, P.; MacKenzie, M.J.; O'Connell, A.; Whiston, F.; et al. Phase II trial of capecitabine plus erlotinib versus capecitabine alone in patients with advanced colorectal cancer. Future Oncol. 2017, 13, 777-786. [CrossRef]

14. Vincent, M.; Breadner, D.; Cripps, M.; Jonker, D.; Klimo, P.; Biagi, J.; Lam, W.; O'Connell, A.; Whiston, F.; Stitt, L.; et al. Phase I/II Trial of Dose-Reduced Capecitabine in Elderly Patients with Advanced Colorectal Cancer. Curr. Oncol. 2017, 24, 261-268. [CrossRef]

15. Hryniuk, W.M. The importance of dose intensity in the outcome of chemotherapy. Important Adv. Oncol. 1988, 4, $121-141$.

16. Nakayama, G.; Tanaka, C.; Uehara, K.; Mashita, N.; Hayashi, N.; Kobayashi, D.; Kanda, M.; Yamada, S.; Fujii, T.; Sugimoto, H.; et al. The impact of dose/time modification in irinotecan- and oxaliplatin-based chemotherapies on outcomes in metastatic colorectal cancer. Cancer Chemother. Pharmacol. 2014, 73, 847-855. [CrossRef] [PubMed]

17. Longo, D.L.; Duffey, P.; DeVita, V., Jr.; Wesley, M.; Hubbard, S.; Young, R. The calculation of actual or received dose intensity: A comparison of published methods. J. Clin. Oncol. 1991, 9, 2042-2051. [CrossRef] [PubMed]

18. Hryniuk, W.; Bush, H. The importance of dose intensity in chemotherapy of metastatic breast cancer. J. Clin. Oncol. 1984, 2, 1281-1288. [CrossRef]

19. Kwak, L.; Halpern, J.; Olshen, A.R.; Horning, S.J. Prognostic significance of actual dose intensity in diffuse large-cell lymphoma: Results of a tree-structured survival analysis. J. Clin. Oncol. 1990, 8, 963-977. [CrossRef] [PubMed]

20. Loibl, S.; Skacel, T.; Nekljudova, V.; Lück, H.J.; Schwenkglenks, M.; Brodowicz, T.; Zielinski, C.; Von Minckwitz, G. Evaluating the impact of Relative Total Dose Intensity (RTDI) on patients' short and long-term outcome in taxane- and anthracycline-based chemotherapy of metastatic breast cancer-A pooled analysis. BMC Cancer 2011, 11, 131. [CrossRef]

21. Luciani, A.; Bertuzzi, C.; Ascione, G.; Di Gennaro, E.; Bozzoni, S.; Zonato, S.; Ferrari, D.; Foa, P. Dose intensity correlate with survival in elderly patients treated with chemotherapy for advanced non-small cell lung cancer. Lung Cancer 2009, 66, 94-96. [CrossRef]

22. Lyman, G.H. Impact of Chemotherapy Dose Intensity on Cancer Patient Outcomes. J. Natl. Compr. Cancer Netw. 2009, 7, 99-108. [CrossRef]

23. Aspinall, S.L.; Good, C.B.; Zhao, X.; Cunningham, F.E.; Heron, B.B.; Geraci, M.; Passero, V.; Stone, R.A.; Smith, K.J.; Rogers, R.; et al. Adjuvant chemotherapy for stage III colon cancer: Relative dose intensity and survival among veterans. $B M C$ Cancer 2015, 15, 62. [CrossRef] [PubMed]

24. Ho, J.; Gill, S.; Woods, R.; Kennecke, H.F. Association of survival outcomes with dose intensity of adjuvant therapy (AT) with capecitabine for colorectal cancer (CRC). J. Clin. Oncol. 2010, 28, 3624. [CrossRef]

25. Grothey, A.; Sobrero, A.F.; Shields, A.F.; Yoshino, T.; Paul, J.; Taieb, J.; Souglakos, J.; Shi, Q.; Kerr, R.; Labianca, R.; et al. Duration of Adjuvant Chemotherapy for Stage III Colon Cancer. N. Engl. J. Med. 2018, 378, 1177-1188. [CrossRef] [PubMed] 
26. Yoshino, T.; Yamanaka, T.; Oki, E.; Kotaka, M.; Manaka, D.; Eto, T.; Hasegawa, J.; Takagane, A.; Nakamura, M.; Kato, T. Efficacy and long-term peripheral sensory neuropathy of 3 vs. 6 months of oxaliplatin-based adjuvant chemotherapy for colon cancer: The ACHIEVE phase 3 randomized clinical trial. JAMA Oncol. 2019, 5, 1574-1581. [CrossRef]

27. Schmoll, H.-J.; Twelves, C.; Sun, W.; O'Connell, M.J.; Cartwright, T.; McKenna, E.; Saif, M.; Lee, S.; Yothers, G.; Haller, D. Effect of adjuvant capecitabine or fluorouracil, with or without oxaliplatin, on survival outcomes in stage III colon cancer and the effect of oxaliplatin on post-relapse survival: A pooled analysis of individual patient data from four randomised controlled trials. Lancet Oncol. 2014, 15, 1481-1492. [PubMed] 